\title{
Icariin as a new potential drug in Alzheimer disease treatment - a review
}

\author{
Patryk Piotr Jasielski ${ }^{1, A, C-D \oplus}$, Faustyna Piędel ${ }^{1, B-D \oplus}{ }^{\text {, }}$, Véronique Petit ${ }^{2, D-F}{ }^{\oplus}$, Konrad Rejdak $^{2, E-F} \oplus$ \\ 'Students' Scientific Society of Neurology, Medical University, Lublin, Poland \\ ${ }^{2}$ Department of Neurology, Medical University, Lublin, Poland \\ A - Research concept and design, B - Collection and/or assembly of data, C - Data analysis and interpretation, \\ $D$ - Writing the article, E - Critical revision of the article, F- Final approval of article
}

\begin{abstract}
Jasielski P, Piędel F, Petit V, Rejdak K. Icariin as a new potential drug in Alzheimer disease treatment - a review. J Pre-Clin Clin Res. $2019 ; 13(4)$ : 167-169. DOI: $10.26444 /$ jpccr/114763
\end{abstract}

\begin{abstract}
Introduction. Alzheimer disease (AD) is one of the best-known diseases. It is neurodegenerative disease characterized by gradual memory loss and dysfunction of behaviour. The pathogenesis of this disease is unclear. Icariin (ICA) is a flavonoid found in a Chinese medicinal herb. It is recognised for a wide range of biological and medical activities: anti-tumour and anti-inflammatory, and also has an impact on the nervous system: stimulates neuroproliferation and prevents neuron's apoptosis. ICA may have a potential role in AD disease treatment.

Objective. The purpose of this article is to review current knowledge about mechanisms and use of ICA in AD treatment. State of knowledge. AD does not present established pathomechanism, about which there are some hypotheses. Each hypothesis mentioned and checked in this review, with result that ICA may have a potential role. One well-known hypothesis is that about amylolytic which is associated with amyloid precursor protein (APP) gene mutation, which leads to amyloid beta $(A \beta)$ protein accumulation and to occurenc of the disease. In this hypothesis, ICA may inhibit $A \beta$ protein aggregation or alter APP expression. ICA may stifle neuronal apoptosis and promote neurogenesis and neuromodulation. According to other hypotheses, ICA could also impact on iron overload in the brain, harmful for neurons hyperhomocysteinaemia, disorder of the intracellular calcium management. Modification of theTau protein structure is another one theory of ICA action. Flavonoid from China may also have an influence on axonal transport.

Conclusions. According to the literature there is no single mechanism of ICA action in slowing down AD progress. A wide range of therapeutic points is demonstrated by the broad effect of this substance. Further research is mandatory.
\end{abstract}

\section{Key words}

Alzheimer disease; Icariin; amyloid precursor protein, neuroprotective effect

\section{INTRODUCTION}

Alzheimer disease $(\mathrm{AD})$ is a neurodegenerative, progressive disease and is the most common cause of dementia worldwide. $\mathrm{AD}$ is characterized by gradual memory loss, devolution of higher cognitive function and changesin behaviour [1]. The pathogenesis of tes disease is unclear. There are different theories: amyloid theory, autophagy, iron overload and many more. Despite sustained research, AD is still untreatable - contemporary treatment only delays the occurence of symptoms and moderates them [2], which is why research about new drugs is so important.

Icariin (ICA) is a major flavonoid constituent found in the Chinese medicinal herb Epimedium brevicornum, which has a wide range of biological and pharmacological properties, including: anti-tumour, estrogenic, anti-inflammatory and antioxidant activity. ICA also has an impact on agedependent disease states, including bone loss, cardiovascular disease, and neurodegenerative disorders. This flavonoid may be useful in AD treatment; it can boost learning and memory abilities. ICA stimulates neuroproliferation and prevents neuron apoptosis $[2,3]$.

Address for correspondence: Patryk Piotr Jasielski, Students' Scientific Society of Neurology, Medical University, Lublin, Poland

E-mail: patryk.jasielski111@gmail.com

Received: 20.10.2019; accepted: 25.11.2019; first published: 31.12.2019

\section{OBJECTIVE}

To review data about the use and mechanisms of the action of ICAs in the treatment of AD.

\section{MATERIALS AND METHOD}

The presented review searched the databases Scopus and Pubmed using the search key words: "Icariin Alzheimer disease". The search criteria included the last 10 years in which the work was published. In addition, only original articles were searched for in the Scopus database. The work was taken into account in if the entire text was freely available. 18 articles were found in Scopus. In the final analysis, 15 articles were selected and 3 were rejected - 2 due to incompatibility with the subject of the review. However, one of the works was double-searched. There were 23 articles in the Pubmed database, 9 of which coincided with the selected Scopus database. Four review papers were rejected. The final review consisted of a total of 25 publications.

Description of the state of knowledge. Since its description in 1906, AD has not yet been stablished regarding its pathomechanism. Not knowing the cause of the disease leads to the use of symptomatic treatment only. Progressive, untreatable $\mathrm{AD}$ leads to the patient's death. Hence, there is a need for research to find the point of the disease handle, 
and thus new treatment methods. In the course of years, new hypothesis have emerged explaining the occurrence of neurodegenerative changes leading to dementia in people affected by $\mathrm{AD}$.

One of the main hypotheses is the amylotic hypothesis, which assumes that mutations in the amyloid precursor protein (APP) gene lead to the development of AD. The accumulation of amyloid-beta $(A \beta)$ protein leads to the formation of older plaques impairing the functioning of synapses, and thus memory processes [1]. In line with the above hypothesis, the decrease in APP expression and A $\beta$ level may be a therapeutic target for the treatment of AD. Studies are available whose results suggest inhibition of $A \beta$ aggregation by ICA [4, 5]. In research of Li F et al., ICA has instigated the improvement of memory function, as well as reducing A $\beta$ and APP levels in the brain [3]. A similar conclusion was made by Zhang ZY et al., assessing the effect of Icariin onan animal model of cerebral amyloidosis for AD in transgenic APP/PS1 mouse [6]. In the study by Zhang, L. et al., in addition, there was a reduction in $\beta$-amyloid burden, and a decrease in APP, and beta-site amyloid precursor protein cleaving enzyme 1 (BACE-1) expression was observed in the transgenic mouse APP model of AD [7].

The above results suggest that the use of ICA may slow the progression of $\mathrm{AD}$. The mechanisms of neuroprotective activity of Icariin are being sought for.

Sheng, C. et al. in the rat AD model induced by A $\beta 1-42$ injection, evaluated the neuroprotective effect of ICA [2]. In seeking the mechanisms for a possible neuroprotective effect of ICA, the researchers benefited from the hypothesis that brain-derived neurotrophic factor (BDNF) tyrosine kinase B (TrkB), protein kinase B (Akt) - BDNF/ TrkB/ Akt pathway, plays an important role in synaptic plasticity $[8$, 9]. After injection of $A \beta 1-42$, rats exhibited memory and spatial orientation deficits in the Morris Water Labyrinth Test. Intragastric administration of ICA caused a decrease in A $\beta 1-42$ activity; in addition, an increase in the number of synapses and restoration of their structure was observed. The results of this study suggest that ICA has an effect on synaptic plasticity through the BDNF/ TrkB/ Akt pathway, thereby acting neuroprotectively. Dongdong Zhang et al. undertook a search for the mechanisms of ICA action in preventing $A \beta$ induced apoptosis. In a study of cultured phochochocytocyte PC12 rats, the researchers showed that administration of $A \beta$ $25-35$ reduced their viability and increased apoptosis. ICA was found to reduce the effects of $A \beta 25-35$ in these cells by inhibiting apoptosis by activating PI3K/Akt signaling [10]. Of interest are the results of the studies by $\mathrm{Li} \mathrm{L}$ and all, which showed that $A \beta$ could have an adverse effect on neurons through a disorder of the intracellular calcium management; however, ICA can restore calcium haemostasis [11]. Nevertheless, Nie J et al. suggest the suppression by ICA of the beta-secretase expression, which results in minimal production of $A \beta$ fragments [12].

Another hypothesis in the pathogenesis $\mathrm{AD}$ concerns tau protein (MAPT) which acts as a stabilisation function for microtubules. Tau protein, with improper construction and function, is the cause of disturbances of the axonal transport. The excess $A \beta$ leads to hyperphosphorylation of the MAPT, thereby postponing it in the form of neurofibrineric degeneration and neuronal death [1]. ICA has been shown to inhibit the hyperphosphorylation of tau proteins [13].
In the search for the mechanisms by which ICA can prevent the disturbance of axonal transport, Yijing Chen et al. obtained interesting results in primary hippocampal cultures from triple-transgenic $(3 \times \beta \mathrm{Tg}) \mathrm{AD}$ mice. It was shown that ICA can promote mitochondrial transport and protect it from fragmentation, which in patients with $A D$ could preserve axonal transport [14].

There are also other potential mechanisms for AD development and study evaluating the impact of ICA on their course. It has been suggested that excitotoxicity is associated with the onset of $\mathrm{AD}$ which may prevent ICA [15]. ICA may reduce iron overload in the brain, which can potentially affect the occurrence of AD [16,17]. Another of the neuroprotective effects of ICA is to protect neurons from the harmful effects of hyperhomocysteinaemia [18]. In addition, there are reports that Icariin improves neurogenesis and the proliferation of neuronal stem cells in the hippocampus [3, 19]. It is surprising is that ICA can improve memory function by stimulating NO/ cGMP signaling and the induction of nitric oxide synthase (NOS) isoforms [20]. In another analysis of computational functions and memory after ICA termination, a simultaneous improvement in cAMP response element-binding protein (CREB) phosphorylation in hippocampal neurons was observed [21].

Currently, the drugs approved by the FDA in the AD analysis are acetylcholinesterase (AChE) inhibitors. The study of Li Y et al. showed an inhibitory effect of ICA on AChE [22].

The latest research shows that ICA can exhibit neuroprotective effects by regulating the autophagy process [23]. The latest results of $\mathrm{Li}, \mathrm{F}$ et al. show a new test point, which is the protective attenuation of endoplasmic reticulum (ER) stress signalling by ICA [24]. ICA can modulate the immune-inflammatory response associated with CD4 $+\mathrm{T}$ cells, and this could inhibit AD progression [25].

\section{CONCLUSIONS}

AD incurability requires research about new substances that might be used in treatment. This involves searching for the pathomechanism of $\mathrm{AD}$ and mechanisms of new drugs action.

The available literature indicates that there is no single mechanism of action for ICA in slowing down AD progress. A wide range of therapeutic points is demonstrated by the broad effect of this substance. There is certainly a need for further research, including those with $\mathrm{AD}$ to evaluate the action of ICA, depending on the dose of the substance and the severity of the disease. More and more recent research results show that ICA can become a drug of the future in the treatment of $\mathrm{AD}$.

\section{REFERENCES}

1. Gaweł M, Potulska-Chromik A. "Choroby neurodegeneracyjne: choroba Alzheimera i Parkinsona” Postępy Nauk Medycznych, 2015; XXVIII(7): $468-476$.

2.Sheng $\mathrm{C}, \mathrm{Xu} \mathrm{P}$, Zhou K, Deng D, Zhang C, Wang Z. "Icariin Attenuates Synaptic and Cognitive Deficits in an $\mathrm{A} \beta_{1-42}$-Induced Rat Model of Alzheimer's Disease". Biomed Res Int. 2017; 7464872. doi: 10.1155/2017/7464872 2017; 2017: 7464872

3. Li F, Dong H. X, Gong Q. H, Wu Q, Jin F, Shi J. S. "Icariin decreases both APP and A $\beta$ levels and increases neurogenesis in the brain of 
Tg2576 mice. "Neuroscience. 2015; 24; 304: 29-35. doi: 10.1016/j. neuroscience.2015.06.010

4. Liu J, Liu Z, Zhang Y, Yin F. "A novel antagonistic role of natural compound icariin on neurotoxicity of amyloid $\beta$ peptide"; Indian J Med Res. 2015: 142(2): 190-195.

5. Chen Y. J, Zheng H. Y, Huang X. X, Han S. X, Zhang D. S, Ni J. Z. et al. "Neuroprotective Effects of Icariin on Brain Metabolism, Mitochondrial Functions, and Cognition in Triple-Transgenic Alzheimer's Disease Mice. "CNS Neurosci Ther. 2016; 22(1): 63-73. doi: 10.1111/cns.12473

6.Zhang ZY, Li C, Zug C, Schluesener HJ. "Icariin ameliorates neuropathological changes, TGF- $\beta 1$ accumulation and behavioral deficits in a mouse model of cerebral amyloidosis". PLoS One. 2014; 9(8): e104616. doi: 10.1371/journal.pone.0104616

7.Zhang L, Shen C, Chu J, Zhang R, Li Y, Li L. "Icariin decreases the expression of APP and BACE-1 and reduces the $\beta$-amyloid burden in an APP transgenic mouse model of Alzheimer's disease”. Int J Biol Sci. 2014; 10(2): 181-191. doi: 10.7150/ijbs.6232

8. Liu H, Xue X, Shi H, Qi L, Gong D. "Osthole upregulates bdnf to enhance adult hippocampal neurogenesis in APP/PS1 transgenic mice". Biological \& Pharmaceutical Bulletin 2015; 38(10): 1439-1449.

9. Nakai N, Nagai T, Tanaka M, Itoh N, Asai N, Enomoto A, et al. "Girdin phosphorylation is crucial for synaptic plasticity and memory: potential role in the interaction of BDNF/TrkB/ Akt signaling with NMDA receptor, "Journal of Neuroscience, 2014: 34(45): 14995-15008.

10.Zhang D, Wang Z, Sheng C, Peng W, Hui S, Gong W, et al.” Icariin Prevents Amyloid Beta-Induced Apoptosis via the PI3K/Akt Pathway in PC-12 Cells". Evid Based Complement Alternat Med. 2015: 235265. doi: 10.1155/2015/235265

11. Li L, Tsai HJ, Li L, Wang XM. "Icariin inhibits the increased inward calcium currents induced by amyloid-beta (25-35) peptide in CA1 pyramidal neurons of neonatal rat hippocampal slice." Am J Chin Med. 2010; 38(1): 113-125.

12. Nie J, Luo Y, Huang XN, Gong QH, Wu Q, Shi JS. “Icariin inhibits betaamyloid peptide segment 25-35 induced expression of beta-secretase in rat hippocampus. Eur J Pharmacol. 2010; 626(2-3): 213-218. doi: 10.1016/j.ejphar.2009.09.039

13. Cui, Z, Sheng Z, Yan X, Cao Z, Tang K. "In silico insight into potential anti-alzheimer's disease mechanisms of icariin" Int J Mol Sci. 2016; 17: 113. doi: 10.3390/ijms170100113

14. Chen YJ, Zheng HY, Huang XX, Han SX, Zhang DS, Ni JZ, et al. ”The protective effect of icariin on mitochondrial transport and distribution in primary hippocampal neurons from $3 \times \mathrm{Tg}-\mathrm{AD}$ mice" CNS Neurosci Ther. 2016; 22(1): 63-73. doi: 10.1111/cns.12473
15.Zong N, Li F, Deng Y, Shi J, Jin F, Gong Q. “Icariin, a major constituent from Epimedium brevicornum, attenuates ibotenic acid-induced excitotoxicity in rat hippocampus." Behav Brain Res. 2016; 313: 111-119. doi: 10.1016/j.bbr.2016.06.055

16.Zhang Y, Kong WN, Chai XQ. "Compound of icariin, astragalus, and puerarin mitigates iron overload in the cerebral cortex of Alzheimer's disease mice", Neural Regeneration ResearchOpen Access. 2018; 13(4): 731-736.

17. Dong XH, Gao WJ, Kong WN, et al. "Neuroprotective effect of the active components of three Chinese herbs on brain iron load in a mouse model of Alzheimer's disease”. Exp Ther Med. 2015; 9(4): 1319-1327. doi: 10.3892/etm.2015.2234

18. Li XA, HO YS, Chen L, Hsiao WL. "The Protective Effects of Icariin against the Homocysteine-Induced Neurotoxicity in the Primary Embryonic Cultures of Rat Cortical Neurons". Molecules. 2016; 21(11): 1557.

19. Fu X, Li S, Zhou S, Wu Q, Jin F, Shi J. "Stimulatory effect of icariin on the proliferation of neural stem cells from rat hippocampus" BMC Complement Altern Med. 2018; 29; 18(1): 34. doi: 10.1186/s12906-0182095-y

20. Jin F, Gong QH, Xu YS, Wang LN, Jin H, Li F, et al. "Icariin, a phoshphodiesterase-5 inhibitor, improves learning and memory in APP/PS1 transgenic mice by stimulation of NO/cGMP signalling" International Journal of Neuropsychopharmacology. 2014; 29(2): 871-881.

21.Zhang Z, Zhang T, Dong K. "Icariin upregulates phosphorylated cyclic adenosine monophosphate response element binding protein levels in the hippocampus of the senescence- accelerated mouse". Neural Regen Res. 2012; 7(12): 885-890. doi: 10.3969/j.issn.1673-5374.2012.12.001

22. Li Y, Zhang XX, Jiang LJ, Yuan L, Cao TT, Li X, et al. "Inhibition of Acetylcholinesterase (AChE): A Potential Therapeutic Target to Treat Alzheimer's Disease." SFChem Biol Drug Des. 2015; 86(4): 776-82. doi: 10.1111/cbdd.12550

23. Chen FJ, Liu B, Wu Q, Liu J, Xu YY, Zhou SY, et al. "Icarin delays brain aging in senescence-accelerated mouse prone 8 (SAMP8) model via inhibiting autophagy". JPET; 2019; 369(1): 121-128. doi: https://doi. org/10.1124/jpet.118.253310

24. Li F, Zhang Y, Lu X, Shi J, Gong Q. "Icariin improves the cognitive function of APP/PS1 mice via suppressing endoplasmic reticulum stress" Life Sciences. 2019; 234(1): 116739.

25.Zhu T, Zhang F, Li H, He Y, Zhang G, Huang N, et al. "Long-term icariin treatment ameliorates cognitive deficits via $\mathrm{CD} 4^{+} \mathrm{T}$ cell-mediated immuno-inflammatory responses in APP/PS1 mice”. Clin Interv Aging. 2019; 14: 817-826. doi: 10.2147/CIA.S208068 